\title{
Estructura y composición florística de la selva mediana subcaducifolia en Hobomó, Campeche, Campeche, México
}

\section{Structure and floristic composition of the medium subdeciduous forest in Hobomó, Campeche, Campeche, Mexico}

\author{
Pedro Zamora-Crescencio,2,2, Roberto Carlos Barrientos-Medina ${ }^{3}$, Alexis Herminio Plasencia-Vázquez², \\ Pascale Villegas², María del Rosario Domínguez-Carrasco², Celso Gutiérrez-Báez,2, Francisco Javier Aguirre-Crespo 4 , \\ Jorge A. Vargas-Contreras ${ }^{4}$, Samuel Uc-Ruvalcava ${ }^{4}$ e Itzel Guadalupe Poot Sarmiento ${ }^{4}$
}

1 Universidad Autónoma de Campeche Herbario UCAM. San Francisco de Campeche, Campeche, México

2 Universidad Autónoma de Campeche. Centro de Investigaciones Históricas y Sociales. Arqueología, Etnohistoria y Ecología Humana. San Francisco de Campeche, Campeche, México.

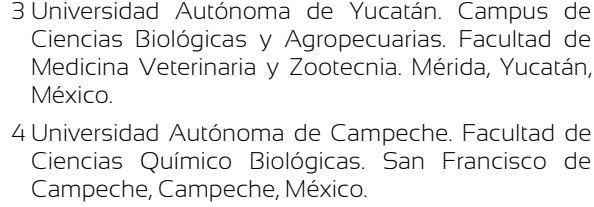
Ciencias Biológicas y Agropecuarias. Facultad de Medicina Veterinaria y Zootecnia. Mérida, Yucatán, México.

4 Universidad Autónoma de Campeche. Facultad de Ciencias Químico Biológicas. San Francisco de Campeche, Campeche, México.

* Autor de correspondencia. pezamora@uacam.mx

\section{RESUMEN}

Se estudió florísticamente la composición y estructura de la selva mediana subcaducifolia en dos sitios del área de Hobomó, Campeche, México. Se seleccionaron dos sitios, en cada uno se delimitaron 10 parcelas de $10 \mathrm{~m}^{2} \times 10 \mathrm{~m}^{2}\left(1000 \mathrm{~m}^{2}\right)$ y se midieron todos los individuos de $\geq 1 \mathrm{~cm}$ de diámetro. El análisis estructural de la selva mediana subcaducifolia se basó en los valores de importancia de las especies. La diversidad se estimó con el índice de Shannon-Wiener ( $\left.H^{\prime}\right)$. Mediante observaciones y recolectas se detectaron 79 especies de plantas vasculares, pertenecientes a 67 géneros y 30 familias. Las familias más diversas fueron Fabaceae y Rubiaceae en ambos sitios. El género más diverso fue Diospyros, seguido de Coccoloba y Guettarda. La diversidad de especies leñosas en los sitios 1 y 2 fue de 3.68 y 3.59, sus valores de equidad (uniformidad) de acuerdo con el índice de Pielou fueron de 0.88 y 0.87 y su densidad fue de 6610 individuos por hectárea (ind/ha) y 8670 ind/ha, respectivamente. Las tres especies con mayor densidad fueron Malpighia glabra, Guettarda combsii y Lonchocarpus xuul en el sitio 1 y Machaonia lindeniana, Coccoloba cozumelensis y Malpighia glabra en el sitio 2. Los valores más altos de los índices de valor de importancia fueron Bursera simaruba, Lonchocarpus xuul, Guettarda combsii, Platymiscium yucatanum y Malpighia glabra en el sitio 1 y Lysiloma latisiliqum, Bursera simaruba, Vitex gaumeri, Lonchocarpus xuul y Machaonia lindeniana en el sitio 2. Estas especies son las más importantes en la estructura y fisonomía de la comunidad vegetal. Se puede concluir que la riqueza de especies arbóreas de los dos sitios estudiados es similar (sitio 1con 63 spp. y sitio 2 con 62 spp.). La escasez o abundancia de las especies está relacionada con la actividad humana (extracción de madera), la cual afecta la estructura y composición florística.

PALABRAS CLAVE: especies leñosas, riqueza, selva, valor de importancia, vegetación.

\section{ABSTRACT}

We studied species the composition and structure in two sites of the subdeciduous tropical forest in Hobomó, Campeche, Mexico. We analyzed 10 plots of $10 \mathrm{~m}^{2} \times 10 \mathrm{~m}^{2}\left(1000 \mathrm{~m}^{2}\right)$, in each site, where all individuals with a diameter $\geq 1 \mathrm{~cm}$ were measured. Vegetation structure was characterized using the importance values of the species, and species diversity was estimated with the Shannon-Wiener (H') Index. A total of 79 species, representing 67 genera and 30 families, was recorded. The most diverse families were Fabaceae and Rubiaceae at the two sites. The most diverse genus was Diospyros, followed by Coccoloba and Guettarda. The diversity of woody species in the study sites were 3.68 and 3.59 in site one and site two, respectively, while their corresponding evenness values (according to Pielou's index) were 0.88 and 0.87 , respectively. The mean densities of the woody species encountered in the sites one and two were $6610 \mathrm{ind} / \mathrm{ha}$ and $8670 \mathrm{ind} / \mathrm{ha}$, respectively. The three species with the greatest density were Malpighia glabra, Guettarda combsii and Lonchocarpus xuul in site one, and Machaonia lindeniana, Coccoloba cozumelensis and Malpighia glabra in site two. The highest values of importance value index were Bursera simaruba, Lonchocarpus xuul, Guettarda combsii, Platymisium yucatanum and Malpighia glabra in site one and Lysiloma latisiliquum, Bursera simaruba, Vitex gaumeri, Lonchocarpus xuul y Machaonia lindeniana in site two. These species are the most important in the structure and physiognomy of the community. We can conclude that the richness of the tree species of the two sites studied its similar (site 1with 63 spp. and site two with 62 spp.). The scarcity or abundance of the species is related to human activity (wood extraction), which affects the structure and floristic composition.

KEYWORDS: wood species, richness, forest, importance value, vegetation. 


\section{INTRODUCCIÓN}

Las selvas tropicales protegen la mayor diversidad biológica del planeta y brindan servicios ecosistémicos fundamentales (Celentano et al., 2011), ya que regulan la atmósfera a nivel global, el sistema hidrológico a nivel local y regional, la escorrentía superficial, la fotosíntesis (Yadav y Gupta, 2006) y con el reciclaje de sus nutrimentos permiten la fertilidad natural del suelo. Además, brindan a las poblaciones rurales y urbanas, maderas útiles en la construcción de viviendas, forraje, combustible, plantas alimenticias y medicinales (Granielo-Vásquez, 1985; Sánchez-Aguilar y Rebollar-Domínguez, 1999; MartínezRamos y Gargía-Orth, 2007).

Los estudios de las comunidades vegetales son fundamentales para el desarrollo de planes de conservación y para hacer un uso sostenible de sus componentes, por lo que su conocimiento, cuantificación y análisis, es conveniente en el contexto actual ante las fuertes presiones antrópicas (Villarreal et al., 2004), tales como la tala clandestina para madera y leña, el crecimiento de tierras para cultivos y los incendios que cada año devastan grandes extensiones de selvas, disminuyendo la mayor parte de las características originales de estas comunidades (Khesoh y Kumar, 2017). Los efectos de estas presiones afectan la estructura de las comunidades vegetales, composición, fisonomía, riqueza de especies (Milchunas y Lauenroth, 1993; Murphy, Lugo, Murphy y Nepstad, 1995; Dunphy, Murphy y Lugo, 2000; Pykälä, 2003; Baquero, Gómez y Orozco, 2011), y la diversidad y distribución (Proulx y Mazumder, 1998). La vegetación es un factor clave en la determinación de la estructura de cualquier ecosistema (Bhatt y Purohit, 2009; Khali y Bhatt, 2014). La pendiente, la altitud, la lluvia y la humedad también juegan un papel importante en la formación de comunidades vegetales y su composición (Kharkwal, Mehrotra, Rawat y Pangtey, 2005).

Para el manejo de las comunidades vegetales, tanto perturbadas como no perturbadas es importante la caracterización cuantitativa de las especies leñosas ya que es un primer paso para entender la estructura de la vegetación, composición y la diversidad (Williams-Linera, 2002; Durán,
Meave, Lott y Segura, 2006; Neelo, Teketay, Kashe y Masamba, 2015). La caracterización es fundamental ya que la distribución de las especies no es homogénea y el status de una especie puede ser rara o abundante o tener restricciones ecológicas por algún factor como el suelo y la humedad (White y Hood, 2004 y Vargas-Rodríguez, Vázquez-García y Williamson, 2005).

Existen trabajos que han contribuido al conocimiento de la composición y estructura de la vegetación en la Península de Yucatán. Entre ellos destacan los de RicoGray (1982); Thien, Bradburn y Welden (1982); Olmsted y Durán (1986); Olmsted y Durán (1990); Rico-Gray et al. (1988 y 1991); White y Darwin (1995); Mizrahi, RamosPrado y Jiménez-Osornio (1997); Sánchez (2000); González-Iturbe, Olmsted y Tun-Dzul (2002); Schultz (2003); White y Hood (2004); Zamora-Crescencio et al. (2007); Leirana-Alcocer, Hernández-Betancourt, SalinasPeba y Guerrero-González (2009); Dupuy et al. (2012); Gutiérrez-Báez, Ortiz-Díaz, Flores-Guido y ZamoraCrescencio (2012a); Navarro-Martínez, Durán-García y Méndez-González (2012); Carreón-Santos y ValdezHernández (2014) y Hernández-Ramírez y García-Méndez (2015). En Campeche, aunque existen trabajos que aportan información sobre estudios cuantitativos de la vegetación (Durán, 1987, 1995; Martínez y Galindo, 2002; DíazGallegos, Castillo-Acosta y García-Gil, 2002; Echeverría, Arreola, Esparza-Olguín, Morales y López, 2014; GarcíaLicona, Esparza-Olguín y Martínez-Romero, 2014; Zamora-Crescencio et al., 2015) pocos estudios han examinado la estructura y composición de la selva mediana subcaducifolia (Gutiérrez-Báez, Zamora-Crescencio y Hernández-Mundo, 2012b; Gutiérrez-Báez, Folan, Folan, Gallegos y Zamora-Crescencio 2017; Dzib-Castillo, Chanatásig-Vaca y González-Valdivia, 2014).

En la Península de Yucatán, la selva mediana subcaducifolia se distribuye en el centro y norte de la península (Flores y Espejel, 1994; Pennington y Sarukhán, 2005) y hacia el sur del municipio de Campeche, su composición florística es característica de cada lugar (Miranda, 1978). Esta selva se caracteriza porque entre 50\% y 75\% de los árboles que la conforman pierden las hojas en 
tiempo de sequía (Flores y Espejel, 1994). La altura de sus árboles oscila entre 10 m y 20 m (Flores y Espejel, 1994). Se presenta en terrenos pedregosos ya sea de ladera o planos con suelo somero. Las especies que caracterizan a la selva mediana subcaducifolia son: Vitex gaumeri, Piscidia piscipula, Enterolobium cyclocarpum, Vachellia pennatula, Caesalpinia gaumeri, Lysiloma latisiliquum, Metopium brownei, Bursera simaruba, Guettarda combsii, Coccoloba cozumelensis, Semialarium mexicanum, Sabal mexicana (Miranda, 1978; Flores y Espejel, 1994). Para el área de estudio, esta comunidad vegetal ha sido afectada por las actividades humanas principalmente para cultivar maíz (lugares planos) y para la extracción de algunas especies como jáabin (Piscidia piscipula) que se utiliza para hacer carbón, el boox káatsim (Senegalia gaumeri) para leña, el k'anchunuup (Thouinia paucidentata) para construcción, para techo de las casas, tasta`b (Guettarda combsii) para construcción de techo de las casas (com. pers. Miguel Mex May).

\section{OBjetIVOS}

El objetivo de este trabajo fue obtener información sobre la estructura y la composición florística de la selva mediana en el ejido de Hobomó, Campeche, que sirva de base para plantear estrategias de protección y conservación del germoplasma local.

\section{MATERIALES Y MÉTODOS}

\section{Área de estudio}

El estudio se desarrolló en el Ejido de Hobomó, localizado en la parte sur del municipio de Campeche, Campeche, México. Se seleccionaron dos sitios separados entre sí por $5.5 \mathrm{~km}$ de distancia. El sitio 1 se encuentra entre las coordenadas $19^{\circ} 37^{\prime} 05^{\prime \prime} \mathrm{N}$ y $90^{\circ} 27^{\prime} 06^{\prime}$ ' W, mientras que el sitio 2 entre $19^{\circ} 35^{\prime} 38^{\prime \prime} \mathrm{N}$ y $90^{\circ} 29^{\prime} 50^{\prime \prime} \mathrm{W}$ (Fig. 1). Su topografía es de lomeríos suaves, el sitio 1 con una altitud de $50 \mathrm{~m} \mathrm{snm}$, mientras que el sitio 2 es de $70 \mathrm{~m} \mathrm{snm}$ (Instituto Nacional de Estadística y Geografía [Inegi], 2000). Las rocas en ambos sitios son sedimentarias del Cenozoico y de formación caliza de edad Eocénica del
Terciario Inferior (Inegi, 2000). El suelo en los dos sitios es de tipo rendzina combinado con litosol, presenta un horizonte mólico, con reacción al $\mathrm{HCl}$; la estructura es granular de tamaño fino y con moderado desarrollo. Con frecuencia presenta pedregosidad con afloramiento de roca madre. Este tipo de suelo es el más productivo, de textura fina arcillosa y asociados a selva mediana subcaducifolia (Inegi, 1984; Flores y Espejel, 1994). El clima de la región es del tipo Aw0, cálido subhúmedo, con precipitación media anual de $1094.1 \mathrm{~mm}$ y temperatura promedio de $27{ }^{\circ} \mathrm{C}$ (Comisión Nacional del Agua [CNA], 2009). Actualmente el uso del suelo en las áreas cercanas a los dos sitios de estudio se utiliza para ganadería caprino y agricultura (cultivo de maíz) a pequeña escala, siendo este último para autoconsumo de la población de Hobomó (com. pers. Carlos Mex May). En el sitio 1 la vegetación es intervenida para extraer algunas especies (Senegalia gaumeri, Thouinia paucidentata, Erythroxylum rotundifolium y Cordia alliodora) para leña y para construcción usan especies como Psidium sartorianum, Caesalpinia sp., Krugiodendron ferreum, entre otras) (com. pers. Carlos Mex May). En el sitio 2 la vegetación es utilizada para colocar apiarios y, afectada por extraer especies para leña (Piscidia piscipula, Thoninia paucidentata, Caesalpinia sp.), especies para construcciones menores (corrales de ganado caprino, cocinas y gallineros) y para hacer carbón utilizan principalmente árboles de jáabin (Piscidia piscipula) (com. pers. Miguel Mex May). Ambos sitios están libres de ganado doméstico.

\section{Procedimiento de muestreo, determinación de materiales y análisis de datos}

Para la selección de los sitios de muestreo se evitaron áreas perturbadas (Parthasarathy, 1999) por el hombre (basadas en presencia/ausencia de tocones) o por causa natural. El muestreo de la vegetación se llevó a cabo en los meses de septiembre y octubre de 2010. En cada sitio de muestreo, en forma aleatoria, se delimitaron 10 parcelas de forma cuadrada de $10 \mathrm{~m} \times 10 \mathrm{~m}\left(100 \mathrm{~m}^{2}\right.$, total $1000 \mathrm{~m}^{2}$; Fig 2), según la metodología propuesta por Gentry (1982) y discutida por Phillips y Miller (2002), que consiste en el 


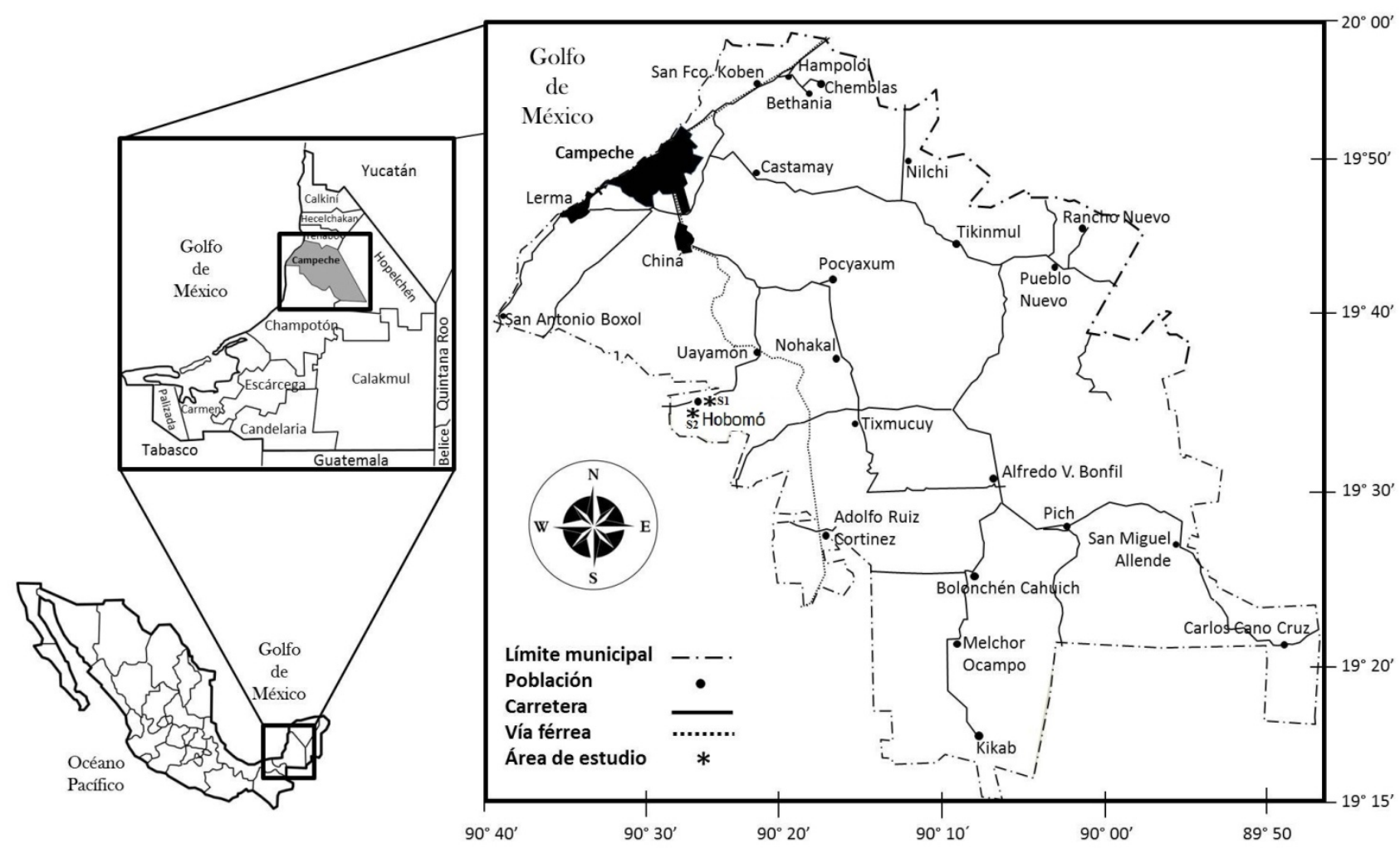

FIGURA 1. Localización del área de estudio.

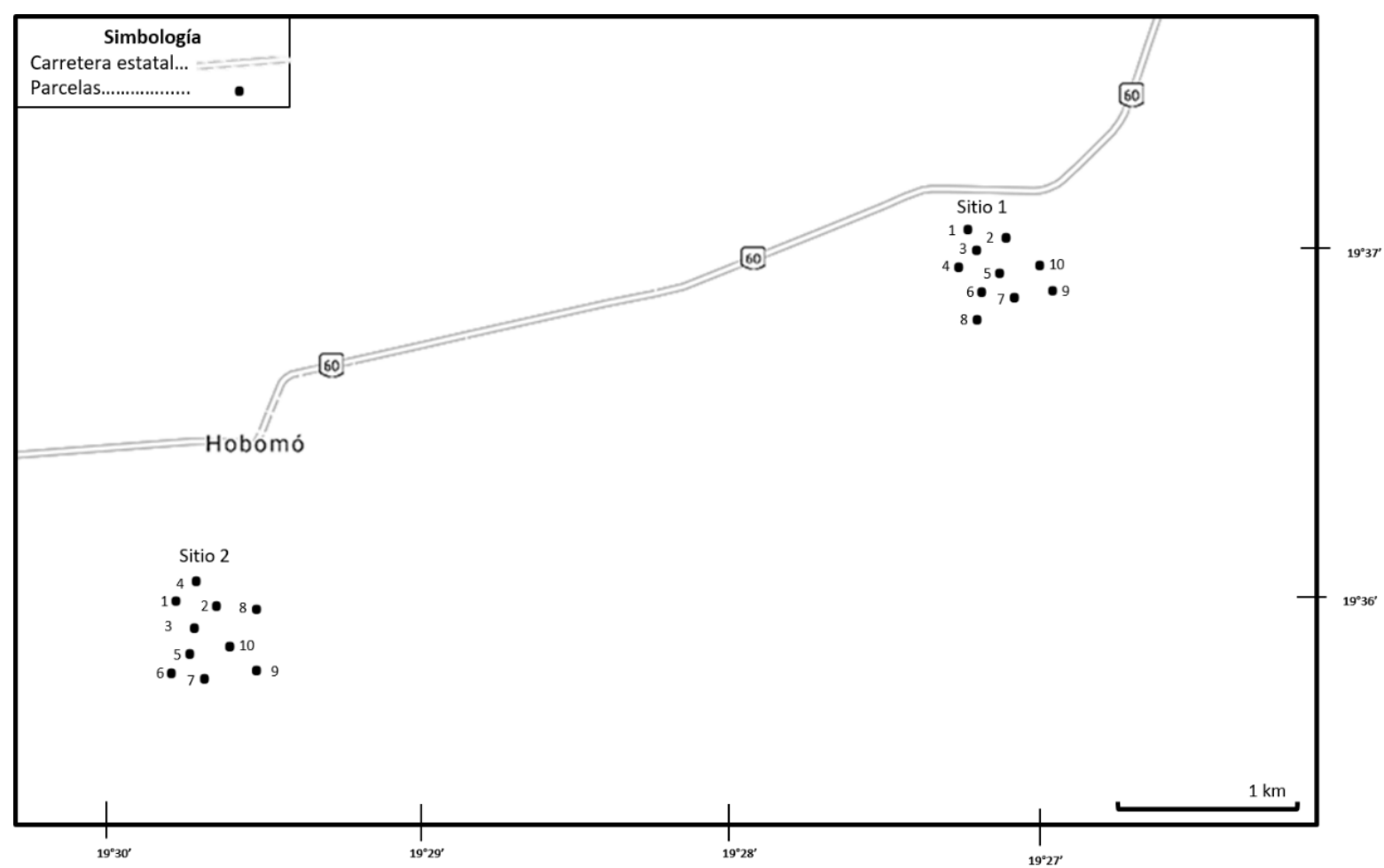

FIGURA 2. Ubicación de las parcelas en los sitios de muestreo. 
levantamiento de la vegetación en unidades de 0.1 ha, metodología utilizada por otros autores (Thomas, 2009, Alvear, Betancur y Francisco-Rosselli, 2010). En cada parcela se consideraron todos los individuos leñosos con diámetros a la altura del pecho $(\mathrm{DAP}=1.3 \mathrm{~m}) \mathrm{DAP} \geq 1$ $\mathrm{cm}$.

La determinación de las especies se hizo in situ, cuando fue posible, o se recolectó material botánico para su determinación mediante comparación con ejemplares depositados en el herbario UCAM y literatura disponible (Standley y Steyermark, 1946, 1949; Ortiz, 1994; Pennington y Sarukhán, 2005; Borhidi, 2006). Los ejemplares fértiles fueron depositados en el herbario (UCAM) del Centro de Investigaciones Históricas y Sociales de la Universidad Autónoma de Campeche, Campeche, México. Posteriormente, otros juegos de ejemplares fueron enviados a herbarios con los que se tiene convenio. La clasificación taxonómica fue efectuada según lo propuesto por el Grupo para la Filogenia de las Angiospermas (APG 2009) y la actualización nomenclatural de las especies por Villaseñor (2016).

El análisis cuantitativo de la estructura de la vegetación leñosa para cada uno de los sitios se basó en el cálculo de la densidad, área basal y frecuencia. Donde la densidad es el número de individuos por área de muestreo, el área basal es la superficie de una sección transversal del tallo del individuo a determinada altura y la frecuencia se refiere a las unidades de muestreo en la cual ocurre una especie (McCune y Grace, 2002). La estructura vertical se analizó utilizando un histograma de frecuencias de alturas para todos los individuos censados. Las distintas clases de diámetro y altura fueron definidas considerando los valores de estos dos parámetros y el número total de individuos. Para obtener el valor de importancia por especie en cada sitio, se calcularon y sumaron la densidad relativa (número de individuos por especie/total de individuos de las especies $\times 100$ ), la frecuencia relativa (número de unidades de muestra en el que se encontró una especie/el número total de unidades de muestra $\times 100$ ) y dominancia relativa (área basal de cada especie/total del área basal de todas las especies $\times 100$ ) (Mueller-Dombois y Ellenberg, 2002). La diversidad alfa se estimó usando el índice de ShannonWiener (H') (Magurran, 1988).

$\mathrm{H}^{\prime}=-\sum p_{i} \ln p_{i}$

Donde:

$P i=$ proporción de la muestra que pertenece a la especie i

La uniformidad o equidad se calculó con el índice de Pielou, el cual se obtiene al dividir el índice de Shannon entre el logaritmo natural del número de especies. Los cálculos se realizaron con el paquete PAST (Hammer, Harper y Ryan, 2001), versión 2.17 .

\section{Resultados}

\section{Composición florística}

En los dos sitios estudiados, se registró un total de 1528 individuos pertenecientes a 79 especies, 67 géneros y 30 familias de plantas leñosas (Suplementos 1 y 2). Fabaceae fue la familia con mayor riqueza (13 especies), seguido de Rubiaceae (10), Ebenaceae, Malvaceae, Polygonaceae y Sapotaceae cada una con cuatro especies, que en conjunto representan $49.4 \%$ de la riqueza de especies registrada en ambos sitios. Las familias con mayor número de géneros fueron Fabaceae (11), Rubiaceae (siete) y Malvaceae (cuatro), aportando 32.8\% del total de los géneros encontrados. Los géneros más ricos fueron Diospyros (cuatro), Coccoloba y Guettarda con tres cada una. Las especies más abundantes fueron Malpighia glabra con 114 individuos, Machaonia lindeniana (109), Coccoloba cozumelensis (84), Lonchocarpus xuul (78), Coccoloba barbadensis (62), Mosannona depressa y Guettarda combsii con 54 cada una, que representaron $36.3 \%$ del total de individuos encontrados en los sitios.

En cuanto al análisis de cada uno de los sitios, se tiene que en el sitio 1 se registró un total de 661 individuos de 63 especies, 54 géneros y 29 familias (Tabla 1). Las familias con mayor número de especies fueron: Fabaceae y Rubiaceae con nueve cada una y Polygonaceae con cuatro. Tres especies incluyeron $19.4 \%$ de los individuos: Malpighia 
glabra con 7.7\% (51 individuos), Guetarda combsii con 6.5\% (43) y Lonchocarpus xuul con 5.1\% (34). En el sitio 2 se encontró un total de 867 individuos agrupados en 62 especies, 55 géneros y 27 familias (Tabla 1). Las familias más diversas fueron: Fabaceae con 12 especies, Rubiaceae (8), Ebenaceae y Polygonaceae con cuatro cada una. Tres especies incluyeron $24.1 \%$ de los individuos: Machaonia lindeniana con 9.5\% (82 individuos), Coccoloba cozumelensis con $7.4 \%$ (64) y Malpigbia glabra con $7.3 \%$ (63). El índice de Shannon mostró que la diversidad de especies del sitio 2 (H' $=3.59)$ es muy similar a la diversidad del sitio $1\left(\mathrm{H}^{\prime}=3.68\right)$, mientras que en términos de la uniformidad ambos sitios tienen valores semejantes ( 0.88 para el sitio 1 y 0.87 para el sitio 2).

\section{Estructura de la vegetación}

Se registraron 15680 ind/ha en los dos sitios, 6610 ind/ha en el sitio 1 y 8670 ind/ha en el sitio 2. Para el sitio 1, Malpigbia glabra fue la especie con mayor densidad (510 ind/ha), seguida de Guettarda combsii (430 ind/ha) y Lonchocarpus xuul (340 ind/ha), que juntas agrupan 19.4\% del total de individuos encontrados. Para el sitio 2, Machaonia lindeniana fue la especie con mayor densidad (820 ind/ha), seguida de Coccoloba cozumelensis (640 ind/ha) y Malpighia glabra (630 ind/ha), representando $24.1 \%$ del total de individuos encontrados. Las diferencias en las densidades de las cinco especies entre los sitios, también se reflejan en los valores totales de área basal. El sitio 1 acumuló $34.13 \mathrm{~m}^{2} /$ ha de área basal, mientras que el sitio 2 fue de $49.27 \mathrm{~m}^{2} /$ ha (Tabla 1$)$.

Con respecto a la estructura vertical por clases de altura, en el sitio 1, 56.1\% de los individuos (371) se localizan en la primera clase ( $1 \mathrm{~m}-4.9 \mathrm{~m})$; mientras que, en el sitio 2, 61.8\% de los individuos (536) están en esta misma clase (Fig. 3). En el sitio 1, las especies representativas que tuvieron mayor proporción de individuos en esta clase (1 $4.9 \mathrm{~m}$ ) de altura fueron Malpighia glabra (46 individuos), Guettarda gaumeri (25), Diospyros anisandra (19) y Mosannona depressa (18). Malpigbia glabra fue una especie común en las primeras tres clases de altura. La última clase $(>13 \mathrm{~m})$ la componen 10 especies y las mejor representadas por abundancia fueron Bursera simaruba, Lonchocarpus xuul, Platymiscium yucatanum y Vitex gaumeri. Las especies mejor representadas en el sitio 2 dentro de la clase de menor altura $(1 \mathrm{~m}-4.9 \mathrm{~m})$ y en función de su abundancia fueron: Machaonia lindeniana (59 individuos), Malpighia glabra (59) y Coccoloba cozumelensis (51). Mientras que la clase de mayor altura (> $13 \mathrm{~m}$ ) estuvo representada por ocho especies y las mejores representadas por sus abundancias fueron Lysiloma latisiliquum, Lonchocarpus xuul, Vitex gaumeri y Bursera simaruba.

TABLA 1. Características de las especies leñosas en los dos sitios de muestreo.

\begin{tabular}{lcc}
\hline Parámetros & Sitio 1 & Sitio 2 \\
\hline Área muestreada (ha) & 0.1 & 0.1 \\
Familias & 29 & 26 \\
Géneros & 54 & 55 \\
Especies & 63 & 62 \\
Ind/ha & 6610 & 8670 \\
AB m²/ha & 34.13 & 49.27 \\
Índice Shannon-Weaner & 3.68 & 3.59 \\
Equitatividad & 0.88 & 0.87 \\
\hline
\end{tabular}




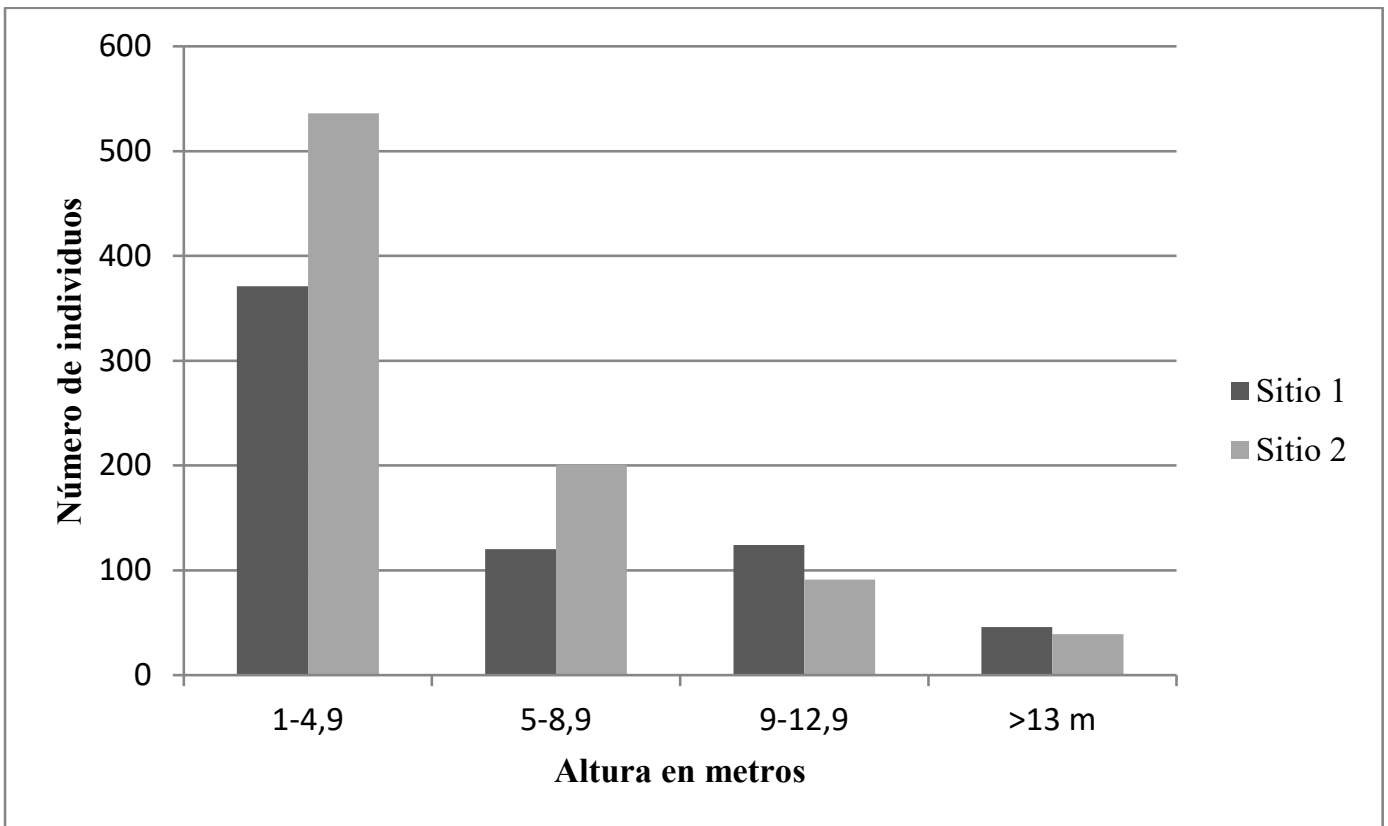

FIGURA 3. Distribución de las clases de altura de los individuos con DAP $\geq 1.0 \mathrm{~cm}$ de los 20 cuadros de selva mediana subcaducifolia en el ejido de Hobomó, Campeche, México.

La representación de la distribución general y por sitio de los individuos en las diferentes clases diamétricas revela una forma de J-invertida, con la mayor frecuencia de individuos en la clase de tamaño pequeño (Fig. 4). El sitio 1 aportó una densidad de 6610 ind/ha con diámetros de $1 \mathrm{~cm}-4.9 \mathrm{~cm}$ (60.5\%), siendo Malpighia glabra, Guettarda gaumeri, Diospyros anisandra, Guettarda combsii y Psidium sartorianum que aportaron una elevada cantidad de individuos $(>20)$ en esta clase, mientras que las especies que alcanzaron la última clase diamétrica $(>29 \mathrm{~cm})$ fueron Bursera simaruba y Lysiloma latisiliquum. El sito 2 está caracterizado por una densidad de $5280 \mathrm{ind} / \mathrm{ha}$ que corresponden a los diámetros de $1 \mathrm{~cm}$ $4.9 \mathrm{~cm}$ (60.9\%), destacan Machaonia lindeniana, Malpighia glabra, Coccoloba cozumelensis, Mosannona depressa y Coccoloba barbadensis al aportar una elevada cantidad de individuos ( $>$ 20) en la primera clase diamétrica, mientras que las especies que alcanzaron la última clase diamétrica $(>29 \mathrm{~cm})$ fueron Vitex gaumeri, Trichilia sp., Bursera simaruba, Coccoloba acapulcensis y Thouinia paucidentata.

En los suplementos 1 y 2 se señalan los componentes y el valor de importancia (VI) de las especies que conforman la estructura de la selva mediana subcaducifolia en los sitios analizados. De las 63 especies registradas en el sitio 1, Bursera simaruba, Lonchocarpus xuul, Guettarda combsii, Platymiscium yucatanum y Malpighia glabra sumaron 28.4\% del VI (Suplemento 1). En el sitio 2, 31.3\% del VI estuvo representado por Lysiloma latisiliqum, Bursera simaruba, Vitex gaumeri, Lonchocarpus xuul y Machaonia lindeniana de las 62 allí registradas (Suplemento 2). Bursera simaruba fue la más importante en el sitio 1 (Suplemento 1), su alto VI está dado por su área basal más que por su densidad y frecuencia colocándola en la primera posición, mientras que en el sitio 2 su VI fue bajo, esto indica que los individuos de Bursera simaruba en este sitio tienen diámetros menores que los individuos del sitio 1, lo que permite suponer que se trata de individuos con mayor edad en el sitio 1. Lysiloma latisiliquum fue la segunda especie en importancia estructural en el sitio 2 (Suplemento 2), su VI está dado por su área basal más que por su densidad y frecuencia relativa, mientras que en el sitio 1 sus parámetros estructurales son bajos reflejándose en su VI más bajo, ocupando la trigésima quinta posición (Suplemento 1). 


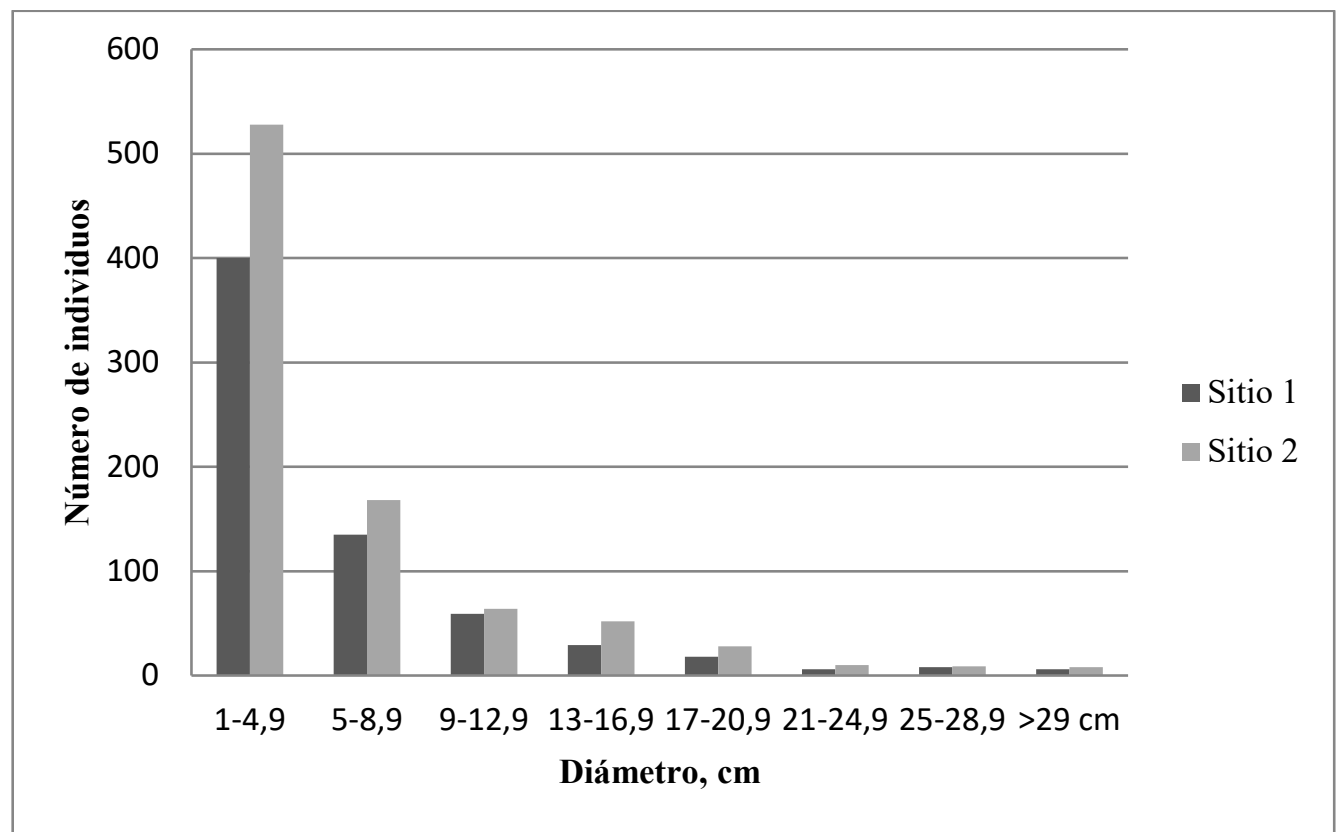

FIGURA 4. Distribución de las clases diamétricas de los individuos con DAP $\geq 1 \mathrm{~cm}$ de los 20 cuadros de selva mediana subcaducifolia en el ejido de Hobomo, Campeche, México.

\section{DISCUSIÓN}

El resultado encontrado en este estudio acerca de la composición florística en ambos sitios estuvo representada por especies de las familias Fabaceae y Rubiaceae, lo cual coincide a los citados por varios autores en selvas medianas subcaducifolias de otros sitios. Por ejemplo, varios autores han registrados que las familias mejor representadas por el número de especies son Fabaceae y Rubiaceae; resultado similar en este estudio (Zamora-Crescencio, García-Gil, Flores-Guido y Ortiz, 2008; Gutiérrez-Báez et al., 2012a; Gutiérrez-Báez et al., 2012b, Escobar, 2013; Echeverría et al., 2014; Dzib-Castillo et al., 2014; García-Licona et al., 2014). El hecho de que la familia Fabaceae sea la mejor representada se debe a que es la más diversa y más ampliamente distribuida en todas las comunidades vegetales presentes en la península de Yucatán (Flores, 2001) y en todo el territorio nacional (Sousa y Delgado, 1998) y mundial (Gentry, 1988, 1992).

En términos de la flora leñosa, comparado con estudios similares (Gutiérrez-Báez et al. 2012; GutiérrezBáez, Zamora-Crescencio y Hernández-Mundo, 2014; Zamora-Crescencio et al., 2008) la riqueza específica de plantas encontradas en este estudio fue mayor. Estas diferencias podrían explicarse a que en este estudio se incluyeron las plantas con DAP $\geq 1 \mathrm{~cm}$. En otros trabajos, Zamora-Crescencio, Gutiérrez-Báez, Villegas, DomínguezCarrasco y Barrientos-Medina (2016); Gutiérrez-Báez et al. (2017), que incluyeron esta misma medida de diámetro, los valores también fueron bajos, lo que puede estar relacionado con la alteración antropogénica de la vegetación en el pasado (Marcelo-Peña, Reynel-Rodríguez, Zevallos-Pollito, Bulnes-Soriana y Pérez-Ojeda del Arco, 2007). Esto muestra que, a pesar de las modificaciones de la selva mediana subcaducifolia en estos sitios sostienen una gran riqueza de especies, por lo que es importante resaltar que estas comunidades vegetales deben manejarse con cautela para no agotar el recurso vegetal local, ya que ofrecen una gran variedad de bienes como leña para cocinar y madera para construir casas y de servicios ambientales como captura de carbono y estabilidad del suelo.

Con relación al índice de diversidad, se observa que no hubo variación en los dos sitios (sitio 1 H' 3.68, 63 spp. y sitio 2 H’ 3.59, 62 spp.), lo que indica que ambos son similares por su riqueza de especies, al igual que por su 
uniformidad o equidad (0.88, 0.87). Sin embargo, comparado con otros estudios de la región con el mismo tipo de vegetación [(Gutiérrez-Báez et al. (2014) (H’ 4.3, 49 spp.), Gutiérrez-Báez et al. (2012) (H’ 4.5, 54 spp.)], la diversidad registrada en este estudio es notablemente baja. Estos valores nos indican que están mayormente relacionados con la distribución de abundancias de las especies más que a su riqueza específica, y no por las condiciones ambientales (Granados-Victorino, SánchezGonzález, Martínez-Cabrera y Octavio-Aguilar, 2017).

La presencia de Machaonia lindeniana, Malpigbia glabra, Coccoloba cozumelensis, Guettarda combsii con altas densidades en este estudio también fueron mencionadas por GutiérrezBáez et al. (2012a ; 2014), sin alcanzar valores importantes para ser consideradas como elementos sobresalientes de la selva mediana subcaducifolia. Así mismo, se registraron para la región de Calakmul a Malpighia glabra, Machaonia lindeniana y Coccoloba cozumelensis, las cuales no destacan por tener bajas densidades y tamaños de diámetros pequeños (Zamora-Crescencio et al., 2012, García-Licona et al., 2014). Mientras especies como Coccoloba cozumelensis y Guettarda combsii con altas densidades también fueron registradas para selvas de los Ejidos de Bethania, Campeche, el Carmen II, Calakmul y Pachuitz, Hopelchen (Zamora-Crescencio et al., 2016, 2018; García-Licona et al., 2014 y Echeverría et al., 2014).

El resultado del análisis de las áreas basales agrupadas en clases diamétricas para ambos sitios refleja que las comunidades vegetales están en pleno crecimiento. Se observa que la estructura por la magnitud de diámetros corresponde a una curva en forma de una J-invertida (Fig. 4), lo que indica que el mayor número de individuos se encuentran en las clases menores. Este patrón de distribución es un ejemplo de una comunidad vegetal en crecimiento (Macario, Torres y Cabrera, 1998), además garantiza la supervivencia de las comunidades vegetales, ya que aquellos árboles eliminados ocasionalmente, serán sustituidos por los individuos en las clases de diámetros menores mediante regeneración natural (León, Vélez y Yepes, 2009; Zamora-Crescencio et al., 2012).
$\mathrm{Al}$ realizar la comparación del valor de importancia relativa (VIR) de los dos sitios, se observó que solo Bursera simaruba y Lonchocarpus xuul se encuentran en ambos sitios entre las primeras cinco especies más importantes: Lochocarpus xuul, Guettarda combsii, Platymiscium yucatanum y Malpighia glabra en el sitio 1 y Bursera simaruba, Vitex gaumeri, Lonchocarpus xuul y Machaonia lindeniana en el sitio 2. Para el sitio 1, la especie con mayor VIR fue Bursera simaruba registrando $28.97 \%$ y para el sitio 2 Lysiloma latisiliquum con $24.83 \%$. Estas especies con altos valores (más de 12\%) de VIR se consideran más importantes ecológicamente que aquellas con valores bajos (Zegeye et al., 2011), debido a sus altos valores de densidad, frecuencia y dominancia.

\section{CONCLUSIONES}

En general, para ambos sitios se registraron 79 especies (9.3\% de la flora vascular estimada para el municipio de Campeche), 67 géneros y 30 familias y a nivel de sitios son similares (sitios 163 spp. y 62 spp. en el sitio 2). Las familias más importantes presentes en la selva mediana subcaducifolia por el número de especies en ambos sitios son Fabaceae, Rubiaceae y Polygonaceae. La vegetación del sitio 1 se encuentra conformada por Malpighia glabra, Guettarda combsii y Lonchocarpus xuul que fueron las más abundantes; en el sitio 2 por Machaonia lindeniana, Coccoloba cozumelensis y Malpighia glabra. La especie con mayor valor de importancia ecológica del sitio 1 fue Bursera simaruba y para el sitio 2 lo fue Lysiloma latisiliquum. Finalmente, se puede decir que la escasez o abundancia de las especies, está relacionada con la actividad humana (extracción de madera), la cual afecta la estructura y composición florística.

\section{RECONOCIMIENTOS}

Este trabajo fue apoyado por el proyecto "Consolidación del herbario (UCAM) del Centro de Investigaciones Históricas y Sociales" que forma parte del proyecto general denominado "Fortalecimiento del Cuerpo Académico Arqueología, Etnohistoria y Ecología Humana: Incremento del Desarrollo Académico y Científico" financiado por FOMIX Campeche-Conacyt (Clave CAMP-2008-93419). Las sugerencias de cambios que hicieron los árbitros 
anónimos para una mejor versión del manuscrito. A los señores Carlos Mex May y Miguel Mex May por darnos información del uso del suelo de las áreas cercanas a los sitios de estudio y del uso de algunas especies. A Yolanda del Socorro Arroyo Dzib y Gabriela Patricia Conic Te por el apoyo en el muestreo de campo.

\section{REFERENCIAS}

Alvear, M., Betancur, J., \& Francisco-Rosselli, P. (2010). Diversidad florística y estructura de remanentes de bosque andino en la zona de amortiguación del parque nacional natural los nevados, cordillera central colombiana. Caldasia, 32, 39-63.

Angiosperm Phylogeny Group [APG. III]. 2009. An update of the Angiosperm Phylogeny Group classification for the orders and families of flowering plants: APG III. Botanical Journal of the Linnean Society, 161, 105-121. doi: 10.1111/j.10958339.2009.00996.x

Baquero, J., Gómez, G., \& Orozco, J. (2011). Composición florística en la sonadora, calarcá Quindío. Armenia. Centro de Estudios e Investigaciones en Biodiversidad y Biotecnología-CIBUQ, Universidad del Quindío.

Bhatt, V.P. \& Purohit, V.K. (2009). Floristic structure and phytodiversity along an elevational gradient in Peepalkoti-Joshimath area of Garhwal Himalaya, India. Nature and Science, 7(9), 63-74.

Borhidi, A. 2006. Rubiáceas de México. Akadémiai Kiadó, Budapest. 512 pp.

Carreón-Santos, R. J. \& Valdez-Hernández, J. I. (2014). Estructura y diversidad arbórea de vegetación secundaria derivada de una selva mediana subperennifolia en Quintana Roo. Revista Chapingo. Serie Ciencias Forestales y del Ambiente, 20(1), 119-130.

Celentano, D., Zahawi, R. A., Finegan, B., Ostertag, R., Cole, R. J., \& Holl, K. D. (2011). Litterfall dynamics under different tropical forest restoration strategies. Biotropica, 43, 279-287. Recuperado de doi: 10.1111/j.1744-7429.2010.00688.x

Comisión Nacional del Agua [CNA] (2009). Base de datos mensuales climatológicos correspondientes a la estación de Campeche. Dirección local Campeche, residencia técnica. Campeche, México.

Díaz-Gallegos, J. R., Castillo-Acosta O., \& García-Gil, G. (2002). Distribución espacial y estructura arbórea de la selva baja subperennifolia en un ejido de la Reserva de la Biosfera Calakmul, Campeche, México. Universidad y Ciencia, 18, 11-28. Recuperado de

http://www.universidadyciencia.ujat.mx/sistema/documentos/ volumenes/18-35-2002/1.pdf
Dunphy, B. K., Murphy, P. G., \& Lugo, A. E. (2000). The tendency for trees to be multiple stemmed in tropical and subtropical dry forests: studies of Guanica forest, Puerto Rico. Tropical Ecology, 41, $161-168$

Dupuy, J. M., Hernández-Stefanoni, J. L., Hernández-Juárez, R. A., Tetetla-Rangel, E., López-Martínez, J. O., Leyequién-Abarca, Tun-Dzul, F. J., \& Pay-Pat, F. (2012). Patterns and correlates of tropical dry forest structure and composition in a highly replicated chronosequence in Yucatán, Mexico. Biotropica, 44(2), 151-162. doi: 10.1111/j.1744-7429.2011.00783.x

Durán-García, R. (1987). Descripción y análisis de la estructura y composición de la vegetación de los petenes del noroeste de Campeche, México. Biótica, 12,181-198.

Durán-García， R. (1995). Diversidad florística de los petenes de Campeche. Acta Botanica Mexicana, 31, 73-84. doi: 10.21829/abm31.1995.740

Durán, E., Meave, J. A., Lott, E. J., \& Segura, G. (2006). Structure and tree diversity patterns at the landscape level in a mexican tropical deciduous forest. Boletin de la Sociedad Botánica de México, 79, 4360.

Dzib-Castillo, B., Chanatásig-Vaca, C., \& González-Valdivia, N. A. (2014). Estructura y composición en dos comunidades arbóreas de la selva baja caducifolia y mediana subcaducifolia en Campeche, México. Revista Mexicana de Biodiversidad, 85, 167-178. doi: org/10.7550/rmb.38706

Echeverría, R. F., Arreola, J., Esparza-Olguín, L. G., Morales, V., \& López, J.J. (2014). Análisis de la composición y estructura de la selva mediana subperennifolia del Ejido Pachuitz, Hopelchén, Campeche, México. Foresta Veracruzana, 16, 1-10.

Escobar, N. (2013). Diagnóstico de la composición florística asociada a actividades agropecuarias en el Cerro Quinini (Colombia). Revista Ciencias Agropecuarias. Universidad de Cundinamarca, 1(1), 10-28.

Flores, J. S. \& Espejel, I. (1994). Tipos de vegetación de la Península de Yucatán. Etnoflora Yucatanense. No. 3. México: Universidad Autónoma de Yucatán.

Flores, J. S. (2001). Leguminosa, florística, etnobotánica y ecología. Etnoflora Yucatanense, No. 18. México: Universidad Autónoma de Yucatán.

García-Licona, J. B., Esparza-Olguín, L. G., \& Martínez-Romero, E. (2014). Estructura y composición de la vegetación leñosa de selvas en diferentes estadios sucesionales en el ejido el Carmen II, Calakmul, México. Polibotánica, 38, 1-26.

Gentry, A. H. (1982). Patterns of neotropical plants species diversity. Evolutionary Biology, 15, 1-84. 
Gentry, A. H. (1988). Changes in plant community diversity and floristic composition on environmental and geographical gradients. Annals of the Missouri Botanical Garden, 75 (1), 1-34. doi: $10.2307 / 2399464$

Gentry, A. H. (1992). Tropical forest biodiversity distributional patterns and their conservational significance. Oikos, 63, 19-28. doi: $102307 / 3545512$

González-Iturbe, J. A., Olmsted, I., \& Tun-Dzul, F. (2002). Tropical dry forest recovery after long term Henequen (sisal, Agave fourcroydes Lem.) plantation in northern Yucatan, Mexico. Forest Ecology and Management, 167, 67-82. doi: 10.1016/S0378-1127(01)00689-2

Granados-Victorino, R. L., Sánchez-González, A., Martínez-Cabrera, D., \& Octavio-Aguilar, P. (2017). Estructura y composición arbórea de tres estadios sucesionales de selva mediana superennifolia del municipio de Huautla, Hidalgo, México, Revista Mexicana de Biodiversidad 88, 122-134. Recuperado de http://www.revista.ib.unam.mx/index.php/bio/article/view/16 $59 / 1425$

Granielo-Vásquez, S. (1985). Uso y abuso de la selva. Los recursos del trópico húmedo. Información Cientifica y Tecnológica, 7, 35-38.

Gutiérrez-Báez, C., Ortiz-Díaz, J. J., Flores-Guido, J. S., \& ZamoraCrescencio, P. (2012a). Diversidad, estructura y composición de las especies leñosas de la selva mediana subcaducifolia del punto de unión territorial (PUT) de Yucatán, México, Polibotánica, 33 , 151-174. Recuperado de https://www.polibotanica.mx/ojs/index.php/polibotanica/artic le/view/883

Gutiérrez-Báez, C., Zamora-Crescencio, P., \& Hernández-Mundo, S.C. (2012b). Estructura y composición florística de la selva mediana subcaducifolia de Mucuychacán, Campeche, México. Foresta Veracuzana, 14(1), 9-16.

Gutiérrez-Báez, C., Zamora-Crescencio, P., \& Hernández-Mundo, S.C. (2014). Estructura y composición florística de la selva mediana subcaducifolia de San Agustín Olá, Campeche, México. Foresta Veracuðana, 16(1), 17-24.

Gutiérrez-Báez, C. Folan, W.J., Folan, L., Gallegos, S., \& ZamoraCrescencio, P. (2017). Estructura y composición florística de la selva mediana subcaducifolia de Imí Campeche, México. Foresta Veracruzana, 19(1), 1-8.

Hammer, Ø., Harper, D. A. T., \& Ryan, P. D. PAST: Paleontological Statistics Software Package for Education and Data Analysis. Palaeontologia Electronica, 1(1), 1-9. Recuperado de https://palaeoelectronica.org/2001 1/past/issue1 01.htm

Hernández-Ramírez, A. M. \& García-Méndez, S. (2015). Diversidad, estructura y regeneración de la selva tropical estacionalmente seca de la Península de Yucatán, México. Revista de Biología Tropical, 63(3),603-616.

Instituto Nacional de Estadística y Geografía [Inegi]. (1984). Carta edafológica. Escala 1:50,000 E15-3. México: Inegi.

Instituto Nacional de Estadística y Geografía [Inegi]. (2000). Carta Topográfica. Escala 1:50,000 E15-B18. México: Inegi.

Instituto Nacional de Estadística y Geografía [Inegi]. (2000). Carta geológica. Escala 1:50,000 E15-3. México: Inegi.

Khali, M. \& Bhatt, V.P. (2014). Community structure of montane forest along the altitudinal gradient in Garhwal Himalaya, India. Journal Ecology \& Natural Environmental., 6(6), 205-214. doi:10.5897//JENE12.093

Kharkwal, G., Mehrotra, P., Rawat, Y. S., \& Pangtey, P.S. (2005). Phytodiversity and growth form in relation two altitudinal gradient in the Central Hymalayan (Kumaun) region of India. Current Science, 89 (5), 873-878. Recuperado de http://www.jstor.org/stable/24111035

Khesoh, P. \& Kumar, H. (2017). Species diversity and community structure of trees and shrubs of japfü mountain, kohima: Nagaland. International Journal of forestry and Crop Improvement, 8(2), 97-105. doi: 10.15740/HAS/IJFCI/8.2/97-105.

Leirana-Alcocer, J. L., Hernández-Betancourt, S., Salinas-Peba, L., \& Guerrero-González, L. (2009). Cambios en la estructura y composición de la vegetación relacionados con los años de abandono de tierras agropecuarias en la selva baja caducifolia espinosa de la reserva de Dzilam, Yucatán. Polibotánica, 27, 53-70.

León, J. D., Vélez, G., \& Yepes, A. P. (2009). Estructura y composición florística de tres robledales en la región norte de la cordillera central de Colombia. Revista Biología Tropical Vol. 57 (4), 11651182.

Macario M., P.A., Torres P., S.A., \& Cabrera C., E.F. (1998). Estructura y composición de una comunidad con Pinus caribaea var. hondurensis (Sénecl.) Barr. y Golf., en el Estado de Quintana Roo, México. Caribbean Journal of Science, 34(1-2), 50-57.

Magurran, A. E. (1988). Ecological Diversity and its Measurement. Princeton: Princeton University Press.

Marcelo-Peña, J. L., Reynel-Rodríguez, C., Zevallos-Pollito, P., BulnesSoriana, F., \& Pérez-Ojeda del Arco, A. (2007). Diversidad, composición florística y endemismos en los bosques estacionalmente secos alterados del distrito de Jaén, Perú. Ecología Aplicada, 6, 9-22.

Martínez, E. \& Galindo-Leal, C. (2002). La vegetación de Calakmul, México: clasificación, descripción y distribución. Boletín Sociedad Botánica México 71, 7-32. 
Martínez-Ramos, M. \& García-Orth, X. (2007). Sucesión ecológica y restauración de las selvas húmedas. Boletín de la Sociedad Botánica de México, 80S, 69-84.

McCune, B., \& Grace, J. B. (2002). Analysis of ecological communities. Estados Unidos: MjM Software Design.

Milchunas, D. G. \& Lauenroth, W. K. (1993). Quantitative effects of grazing on vegetation and soils over a global range of environments. Ecological Monographs, 63, 327-366. doi: $10.2307 / 2937150$

Miranda, F. (1978). Vegetación de la peninsula de Yucatán. Colegio de Postgraduados-SARH. Chapingo, México.

Mizrahi, A., Ramos-Prado, J. M., \& Jiménez-Osornio, J. (1997). Composition, structure and management potential of secondary dry tropical vegetation in two abandoned plantations of Yucatan, Mexico. Forest Ecology and Management, 96 (3), 273-282. doi: 10.1016/S0378-1127(97)00008-X

Muller-Dombois, D. \& Ellemberg, H. (2002). Aims and methods of vegetation ecology. Nueva York, Estados Unidos: John Willer and Sons.

Murphy, P.G., Lugo, A.E., Murphy, A.J., \& Nepstad, D.C. (1995). The dry forests of Puerto Rico's south coast. In Lugo, A.E., Lowe, C. (Eds.), Tropical Forests: Management and Ecology (pp. 178-209). New York: Springer-Verlag Inc.

Navarro-Martínez, A., Durán-García, R., \& Méndez-González, M. (2012). El impacto del huracán Dean sobre la estructura y composición arbórea de un bosque manejado en Quintana Roo, México. Madera y Bosques, 18(1), 57-76. doi: $10.21829 /$ myb.2012.1811138

Neelo, J., Teketay, D., Kashe, K., \& Masamba, W. (2015). Stand structure, diversity and regeneration status of woody species in open and exclosed dry woodland sites around Molapo farming areas of the Okavango Delta, northeastern Botswana. Open Journal of Forestry, 5, 313-328. doi: 10.4236/ojf.2015.54027.

Olmsted, I. \& Durán, R. (1986). Aspectos ecológicos de la selva baja inundable de la Reserva Sian Ka'an, Quintana Roo, México. Biótica, 11(3), 151-179.

Olmsted, I. \& Durán, R. (1990). Vegetación de Sian Ka'an. En L. D. Navarro y J. G. Robinson (Eds.) Diversidad biológica en la Reserva de la Biosfera de Sian Ka'an, Quintana Roo, México. Centro de Investigaciones de Quintana Roo.

Ortiz-Díaz, J. J. 1994. Polygonaceae. Etnoflora Yucatanense, No. 10. México. Universidad Autónoma de Yucatán.

Parthasarathy, N. (1999). Tree diversity and distribution in undisturbed and human-impacted sites of tropical wet evergreen forest in southern Western Ghats, India. Biodiversity and Conservation, 8, 1365-1381. doi: 10.1023/A:100894907385.

Pennington, T. D., \& Sarukhán, J. (2005). Árboles Tropicales de México. Manual para la Identificación de las Principales Especies. Universidad Nacional Autónoma de México-Fondo de Cultura Económica, México, D.F.

Phillips, O. \& Miller, J. (2002). Global patterns of plant diversity: Alwyn H. Gentry's forest transect data set. Monographs in Systematic Botany from the Missouri Botanical Garden St. Louis, Missouri, U.S.A.

Proulx, M. \& Mazumder, A. (1998). Reversal of grazing impact on plant species richness in nutrient-poor vs. nutrient-rich ecosystems. Ecology, 79, 2581-2592. doi: 10.1890/00129658(1998)079[2581:ROGIOP]2.0.CO;2

Pykälä, J. (2003). Effects of restoration with cattle grazing on plant species composition and richness of semi-natural grasslands. Biodiversity and Conservation, 12, 2211-2226. doi: 10.1023/A:1024558617080

Rico-Gray, V. (1982). Estudio de la vegetación de la zona costera inundable del noroeste del estado de Campeche, México: Los Petenes. Biótica, 7, 171-190.

Rico-Gray, V., García-Franco, J.G., Puch, A. \& Sima, P. (1988). Composition and structure of a tropical dry forest in Yucatan, Mexico. International Journal Ecology Environmental Science, 14, 21-29. Recuperado de http://agris.fao.org/agrissearch.do?recordID:US202301672154.

Rico-Gray, V. \& García-Franco, J. G. (1991). The maya and the vegetation of the Yucatan Peninsula. Journal of Ethnobiology, 11,135-142. Recuperado de https://ethnobiology.org/sites/default/files/pdfs/JoE/11$1 / . \mathrm{pdf}$

Sánchez-Aguilar, R. L. \& Rebollar-Domínguez, S. (1999). Deforestación en la Península de Yucatán, los retos que enfrenta. Madera y Bosques, 5(2), 3-17. doi: 10.21829/myb.1999.521344

Sánchez S., O. (2000). Análisis estructural del Jardín Botánico. En O. Sánchez Sánchez, \& G. A. Islebe (Eds.), El Jardín Botánico Dr. Alfredo barrera Marín: fundamentos y estudios particulares. El Colegio de la Frontera Sur y Comisión Nacional para el Conocimiento y Uso de la Biodiversidad.

Sousa S., M. \& Delgado S., A. (1998). Leguminosas mexicanas: fitogeografía, endemismo y orígenes. En T.P. Ramamoorthy, R. Bye, A. Lott, \& J. Fa (Eds.) Diversidad Biológica de México: orígenes y distribución (pp. 449-500). México, D.F.: Universidad Nacional Autónoma de México, 
Schultz, G. P. (2003). Structure and diversity of the Forests at the Edén Ecological Reserve. A. Gómez-Pompa, M. F. Allen, S. L. Fedick, y J. J. Jiménez-Osornio (Eds.). The lowland maya area. Three millennia at the humanwildland interface. (pp. 91-114). CRC Press.

Standley, P. C. \& Steyermark, J. A. 1946. Flora of Guatemala, Part V. Chicago: Chicago Natural History Museum.

Standley, P. C. \& Steyermark, J. A. 1949. Flora of Guatemala, Part VI. Chicago: Chicago Natural History Museum.

Thien, L. B., Bradburn, A. S. \& Welden, A. L. (1982). The woody vegetation of Dzibilchaltum: a mayan archaeological site in northwest, Yucatn, Mexico. Middle American Research Institute Occasional. Papers, 5,1-18.

Thomas, E. (2009). New light on the floristic composition and diversity of indigenous territory and national park Isoboro-Sécure, Bolivia. Biodiversity Conservation, 18, 1847-1878. doi 10.1007/s10531008-9561-7.

Vargas-Rodríguez, Y. L., Vázquez-García, L. A., \& Williamson, G. B (2005). Environmental correlates of tree and seedling-sapling distributions in a Mexican tropical dry forest. Plant Ecology, 180, 117-134.doi: 10.1007/s11258-005-3026-9

Villarreal, H., M. Álvarez, M., Escobar, F., Fagua, G., Mendoza, H., Ospina, M \& Umaña, A.M. (2004). Manual de métodos para el desarrollo de inventarios de biodiversidad. Bogotá, Colombia: Programa de Inventarios de Biodiversidad. Instituto de Investigación de Recursos Biológicos Alexander von Humboldt.

Villaseñor, J. L. (2016). Checklist of the native vascular plants of Mexico. Revista Mexicana de Biodiversidad, 87(3), 559-902. doi: 10.1016/j.rmb.2016.06.017

White, D. A. \& Darwin, S. P. (1995). Woody vegetation of tropical lowland deciduous forest and mayan ruins in the north-central Yucatan Peninsula, Mexico. Tuline Studies in Zoology and Botany, 30, $1-25$.

White, D. A. \& Hood, C. S. (2004). Vegetation patterns and environmental gradients in tropical dry forest of the northern Yucatan Peninsula. Journal of Vegetation Science, 15,151-160. doi: 10.1111/j.1654-1103.2004.tb02250.x

Williams-Linera, G. (2002). Tree species richness complementarity, disturbance and fragmentation in a Mexican tropical montane cloud forest. Biodiversity and Conservation, 11,1825-1843. Recuperado de https://link.springer.com/content/pdf/10.1023\%2FA\%3A1020 346519085.pdf

Yadav, A. S. \& Gupta, S. K. (2006). Effect of micro-environmental and human disturbance on the diversity of woody species in the
Sariska Tiger Project in India. Forest Ecology and Management, 225, 178-189. doi: 10.1016/j.foreco.2005.12.058

Zamora-Crescencio, P. (2007). Caracterización de la vegetación y su manejo en el municipio de Trucacab, Yucatán. Tesis de maestría. Facultad de Medicina Veterinaria y Zootecnia. Universidad Autónoma de Yucatán.

Zamora-Crescencio, P., François Mas, J., Rico-Gray, V., DomínguezCarrasco, M. R., Villegas, P., Gutiérrez-Báez, C., \& BarrientosMedina, R. C. (2015). Composición y estructura arbórea de petenes en la reserva de la biosfera de los petenes, Campeche, México. Polibotánica, 39, 1-19.

Zamora-Crescencio, P., Gutiérrez-Báez, C., Villegas, P., DomínguezCarrasco, M. R., \& Barrientos-Medina, R. C. (2016). Composición y estructura del componente arbóreo de la vegetación secundaria en Campeche, México. Foresta Veracruzana, 18(1), 17-24.

Zamora-Crescencio, P., García-Gil, P. G., Flores-Guido, J. S., \& Ortiz, J. J. (2008). Estructura y composición florística de la selva mediana subcaducifolia en el sur de Yucatán, México. Polibotánica, 26, 33-66.

Zamora-Crescencio, P., Gutiérrez-Báez, C., Folan, W.J., DomínguezCarraszo, Ma. del R., Villegas, P., Cabrera Mis, G., Castro-Angulo, C. M., \& Carballo, J. C. (2012). La vegetación leñosa del sitio arqueológico de Oxpemul, municipio de Calakmul, Campeche, México. Polibotánica, 33, 131-150.

Zamora-Crescencio, P., Rico-Gray, V., Ramírez-Mediana, L. N. G., Barrientos-Mediana, R. C., Plasencia Vázquez, A. H., Villegas, P., Domínguez-Carrasco, M. R., \& Gutiérrez-Báez, C. (2018). Composición y estructura de la vegetación secundaria en Bethania, Campeche, México. Polibotánica, 45, 57-74. doi: 10.18387/polibotánica.45.5

Zegeye, H., Teketay, D., \& Kelbessa, E. (2011). Diversity and regeneration status of woody species in Tara Gedam and Abebaye Forests, Northwestern Ethiopia. Journal of Forestry Research, 22, 315-328. doi: 10.1007/s11676-011-0176-6.

Manuscrito recibido el 28 de noviembre de 2018

Aceptado el 27 de julio de 2019

Publicado el 30 de marzo de 2020

Este documento se debe citar como:

Zamora-Crescencio, P., Barrientos-Medina, R. C., PlasenciaVázquez, A. H., Villegas, P., Domínguez-Carrasco, M. R., 
Gutiérrez-Báez, C., Aguirre-Crespo. F. J., Vargas-Contreras, J. A., Uc-Ruvalcava. S., \& Poot S., I. G. (2020). Estructura y composición florística de la selva mediana subcaducifolia en Hobomó, Campeche, Campeche, México. Madera y Bosques, 26(1), e2611899. doi: 10.21829/myb.2020.2611899.
Madera y Bosques por Instituto de Ecología, A.C. se distribuye bajo una Licencia Creative Commons Atribución-NoComercialCompartirlgual 4.0 Internacional. 